\title{
An experimental and numerical study of micro deep drawing of SUS304 circular cups
}

\author{
Liang Luo ${ }^{1}$, Zhengyi Jiang ${ }^{1, *}$, Dongbin $\mathrm{Wei}^{1,2}$, Ken-Ichi Manabe ${ }^{3}$, Hideki Sato ${ }^{3}$, Xiaofeng $\mathrm{He}^{4}$, \\ and Pengfei $\mathrm{Li}^{4}$ \\ 1 School of Mechanical, Materials \& Mechatronic Engineering, University of Wollongong, Wollongong, NSW 2522, Australia \\ 2 School of Electrical, Mechanical and Mechatronic Systems, University of Technology, Sydney, NSW 2007, Australia \\ 3 School of Science and Engineering, Tokyo Metropolitan University, 1-1 Minami-Osawa, Hachioji-shi, Tokyo 192-0397, Japan \\ 4 School of Mechanical Science and Engineering, Huazhong University of Science and Technology, 1037 Luoyu Road, Wuhan 430074, \\ P.R. China
}

Received 15 September 2015 / Accepted 2 November 2015

\begin{abstract}
Micro deep drawing is a promising technology for mass production of complex 3D micro metal products. Significant size effects at a micro scale, however, obstruct application of this technology and block utilisation of traditional finite element method (FEM). Therefore, a micro tensile test system was developed to obtain accurate material properties considering size effects. Subsequently, a Voronoi blank model was developed for the micro scale simulation. Moreover, micro deep drawing experiments were conducted and their results were compared with the simulation results. The simulation results have a good agreement with the experimental data. Furthermore, the wrinkling at the cup mouth increases with the growth of grain sizes on the SUS304 sheets.
\end{abstract}

Key words: Micro deep drawing, Micro tensile tests, Size effects, Voronoi, FEM

\section{Introduction}

Micro forming technologies have drawn global attentions with consideration of an industrial miniaturisation trend [1]. Compared to other micro manufacturing methods, micro forming technologies have obvious advantages, such as the capacity to handle metals, the potential of mass production and the ability to produce complex 3D structures. Micro deep drawing (MDD), an important micro forming technology, shares all the superiorities of the micro forming technologies while faces problems introduced by reduced sizes. Regarding the machining system, the difficulties with positioning and transporting of raw material in the drawing process arise due to small sizes. Correspondingly, a combined blanking-drawing process was developed and realised by researchers [2]. Therefore, preparation of the raw material for the MDD and performance of the MDD can be conducted within one stroke. However, the precision requirement on both the machining system and the products is another issue in the micro forming [3]. Firstly, a high accuracy in the machine is essential considering the minute sizes of the micro products [4]. Secondly, product's measurement and the raw material

\footnotetext{
*e-mail: jiang@uow.edu.au
}

properties measurement need adjustments for small sizes and precision request. Furthermore, due to the change of deformation behaviour introduced by the tiny sample sizes, traditional machine design methods cannot be utilised to manufacture high quality products. Therefore, the FEM, as a useful aid to experiments, is important to investigate and optimise the micro forming processes.

Because of the size effects, the normal FEM cannot be directly applied in a micro scale simulation. In terms of material at the micro scale, significant changes in material properties were observed by researchers [5-8]. With a decrease of the sample size, material flow stress decreases. Correspondingly, the new models, such as the surface layer model [9] and the modified surface layer model [10], were carried out as explanations. Due to the increase proportion of the surface layer where the dislocation is less restricted and deformation resistance is lower than that of the inner part, the material flow stress decreases with a decrease of the sample size. Moreover, scatter of experimental data increases with a decrease of the sample size. With a few grains on the characteristic direction of the samples, influences of individual grains become significant, and therefore samples containing a few grains exhibit obvious data scatter in the experiments. 


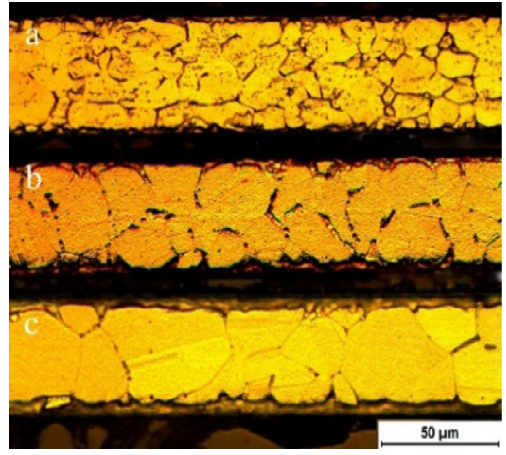

Figure 1. Microstructures of sheets heated under different temperatures (a) $975{ }^{\circ} \mathrm{C}$, (b) $1050{ }^{\circ} \mathrm{C}$ and (c) $1100{ }^{\circ} \mathrm{C}$.

In this study, stainless steel SUS304 sheets with different heat treatments were chosen for the micro deep drawing experiments, and a micro tensile test system was developed to obtain mechanical properties of the sheets including the size effects. Consequently, a Voronoi model was developed based on the real microstructures of the SUS304 sheets utilising the material parameters obtained from the micro tensile tests $[11,12]$. Finally, the simulation and experimental results were compared and discussed.

\section{Experimental}

\subsection{Heat treatment}

Stainless steel SUS304 sheets with a thickness of $50 \mu \mathrm{m}$ were heated at different temperatures under an argon gas protection atmosphere, and microstructures of these sheets were observed under a metallurgical microscope. Based on comparison with the grains sizes and shapes of these differently treated sheets, three heat treatments at temperatures of 975, 1050 and $1100{ }^{\circ} \mathrm{C}$ for $2 \mathrm{~min}$ respectively were selected. Therefore, the grains on these treated sheets were of equiaxed crystals and their average grain sizes were 10,20 and $40 \mu \mathrm{m}$ respectively, as shown in Figure 1. Based on the heating temperatures, sheets were classified as material groups 1-3 correspondingly. Table 1 shows the classification of each material group and their general grain sizes.

\subsection{Micro tensile tests}

Tensile tests were then conducted to obtain mechanical properties of each material group for finite element simulation. Taking the size effects into account, tensile test samples were scaled down, and therefore the width of each sample was only $1 \mathrm{~mm}$, as shown in Figure 2a. Correspondingly, a micro tensile test system was developed, as shown in Figure 2b. Due to the small sizes, traditional elongation measurements cannot be used. Therefore, an image processing program was developed in MATLAB to calculate the strain as a non-contact elongation measurement. A parallel area on the middle of the tensile samples was firstly marked with a contrasting colour to the tensile samples. A video focusing on this area was recorded by the
Table 1. Heating temperatures and average grain sizes after heat treatments.

\begin{tabular}{cccc}
\hline $\begin{array}{c}\text { Group } \\
\left({ }^{\circ} \mathrm{C}\right)\end{array}$ & $\begin{array}{c}\text { Heating temperature } \\
(\mathrm{min})\end{array}$ & $\begin{array}{c}\text { Heating time } \\
(\mu \mathrm{m})\end{array}$ \\
\hline 1 & 975 & 2 & 10 \\
2 & 1050 & 2 & 20 \\
3 & 1100 & 2 & 40 \\
\hline
\end{tabular}

(a)

(b)
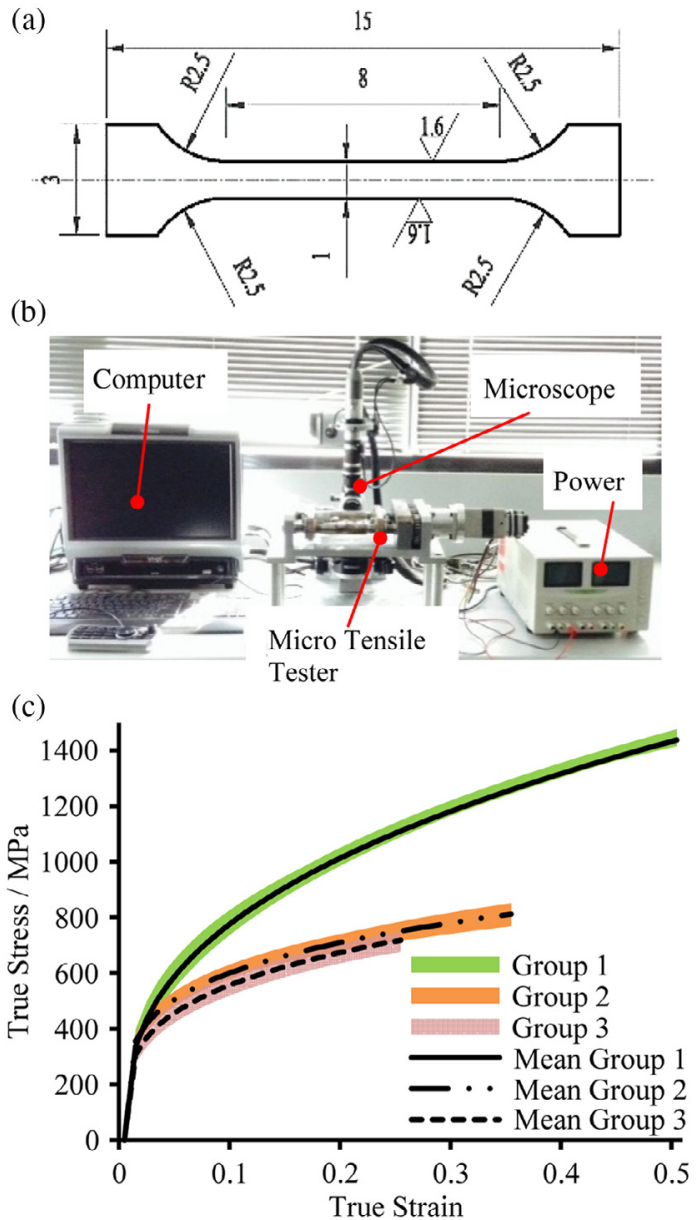

Figure 2. (a) Tensile sample, (b) micro tensile test system and (c) strain-stress curves of each material group.

microscope and then separated to individual images by MATLAB. Subsequently, the colourful images were transferred to grey level images and then to binary images. During the transformation, noise reduction process was conducted to eliminate small areas where the marked colour was lost during the tensile tests. Therefore, errors introduced by the colour loss in the marketed area during the tensile tests can be eliminated. Consequently, boundaries of the marked area on the micro tensile samples can be obtained through colour value comparison between adjacent pixels. If the colour values, being zero or one for a pixel in the binary image, of neighbouring pixels are different, the pixel was selected as a boundary point. Once all boundary points were found, the points were sorted according to their $x$ and $y$ coordinates sequentially. Thus four 
Table 2. Parameters of materials' properties.

\begin{tabular}{lccc}
\hline Group & Strength coefficient $K(\mathrm{MPa})$ & Strain hardening index $n$ & Elastic modulus $E(\mathrm{GPa})$ \\
\hline 1 & $1771 \sim 1908$ & $0.324 \sim 0.405$ & $189.0 \sim 220.0$ \\
2 & $959.3 \sim 1073$ & $0.212 \sim 0.249$ & $185.7 \sim 221.7$ \\
3 & $957.1 \sim 1068$ & $0.227 \sim 0.294$ & $183.3 \sim 216.9$ \\
\hline
\end{tabular}

(a)

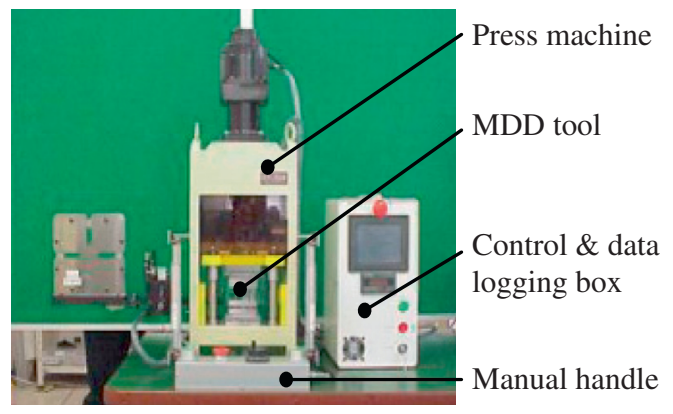

(b)

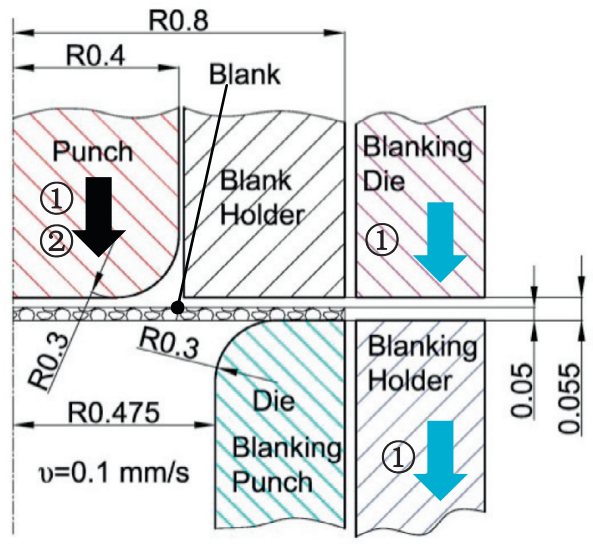

Figure 3. (a) Micro deep drawing system and (b) MDD drawing process sketch.

groups of points can be divided as four boundaries of the marked area. The length, along the length direction of the tensile sample, of the marked area can then be calculated based on these boundaries. By comparing the length of the marked area in each image, the strain can be obtained. Furthermore, the real length of the marked area can be obtained via a scale factor from the scale bar on the image. The scale factor is the ratio of the number of pixels that a scale bar has to the displayed length value on the scale bar. Simultaneously, a force sensor recorded and transferred the tensile force signal to the computer. By matching the video and the force recording frequencies, both engineer and true strain-stress curves can be obtained.

Figure $2 \mathrm{c}$ displays the true strain stress curves covering 10 repeated tests and their mean value of each material group. Table 2 lists the parameters of strain stress curves fitted with the power law equation. With a low heating temperature, grains were small and the flow stress was high. That is because that the annealing effect, which eliminates the work hardening of the raw sheets, was weak at low-temperature heating condition. Moreover, small grain size means large quantity of grain boundaries where dislocation movement is blocked and dislocation piles up. The accumulation of dislocation thus increased the deformation resistance which is presented as the tensile strength in the tensile tests. Therefore, tensile strength of the material group 1 was the highest among all material groups. Moreover, with a few grains in the thickness direction, randomness of each grain's properties significantly impacts the overall properties of the samples and leads to great scatter of samples' properties. The total elongation also decreased with the increase of grain size as a few grains in thickness direction caused fast development of micro cracks and thus early fracture of tensile samples.

\subsection{Micro deep drawing}

The micro deep drawing experiments have been conducted on a MDD system. Figure 3a displays the whole MDD system, and Figure $3 \mathrm{~b}$ illustrates the key parameters of the MDD machine and process setting. Firstly, the blanking die and the blanking holder moved downwards at a speed of $0.1 \mathrm{~mm} / \mathrm{s}$ and the die stayed still as a blanking punch. Therefore, a raw blank for the following drawing process was cut at the first half stroke. Subsequently, the punch moved down continuously and contacted with the blank, whereas the die stayed still. Finally, a micro circular cup was drawn by the punch at the end of the second half stroke. Due to this design of the MDD system, the press machine performing one stroke can fulfil the blanking and the micro deep drawing processes subsequently.

The drawing forces obtained from 10 experiments for each material group were recorded, and their average values are shown in Figure 4. The drawing force had a relatively slow increase initially, and a fast yet constant increase until reaching a peak value. After remaining this peak value for a short period, drawing force of each material group decreased to a nonzero value at the end of the process. Initially, the resistance of bending dominated the drawing force, while other forces were small. As the process continued, large deformation caused high flow stress, and simultaneously friction force increased due to increasing contact forces. Hence, the drawing force increased significantly during the following period. Furthermore, with smaller elastic module, material group 1 had slower growth of drawing force initially, whereas in the later period, the higher strain hardening rate increased flow stress significantly, resulting in the faster growth of drawing force compared with that of the other two material groups. Finally, the peak drawing force was the highest for the material group 1 among all 


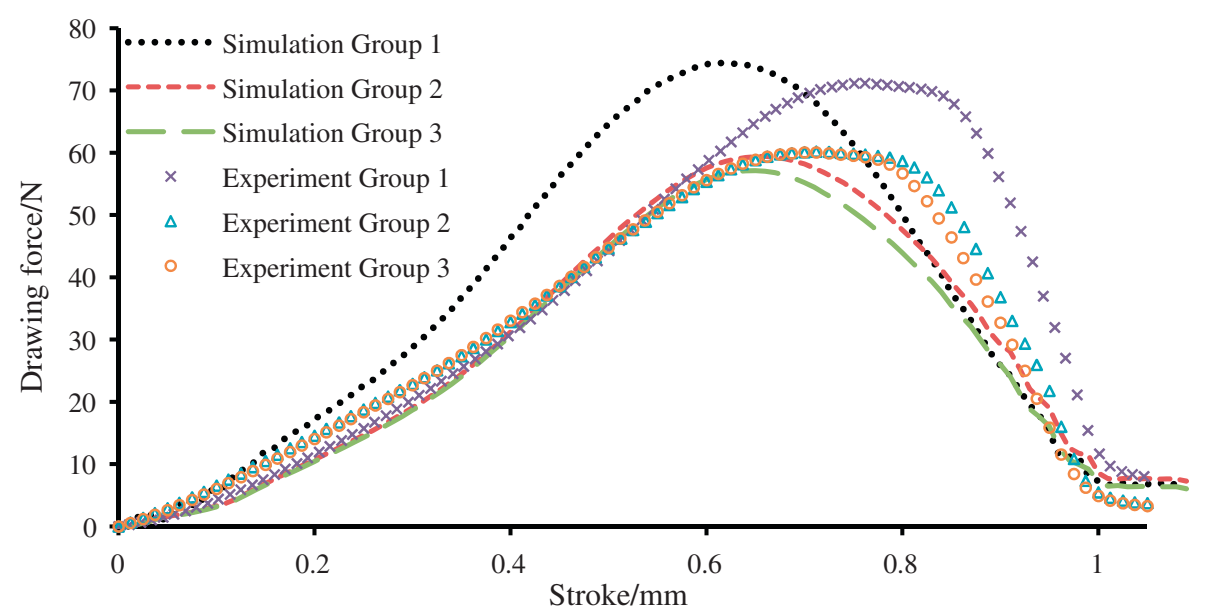

Figure 4. Drawing forces in simulation and experiments.

Table 3. Key parameters of the formed cups for wrinkling measurement.

\begin{tabular}{|c|c|c|c|c|c|c|}
\hline Group & Min inner diameter $(\mu \mathrm{m})$ & Max inner diameter $(\mu \mathrm{m})$ & Outer diameter $(\mu \mathrm{m})$ & Max distance $(\mu \mathrm{m})$ & Min distance $(\mu \mathrm{m})$ & Wrinkles $(\%)$ \\
\hline 1 & 787.083 & 816.645 & 941.092 & 77.004 & 62.223 & 24.546 \\
\hline 2 & 780.558 & 814.277 & 938.137 & 78.789 & 61.930 & 27.334 \\
\hline 3 & 789.532 & 831.042 & 950.033 & 80.251 & 59.496 & 35.091 \\
\hline
\end{tabular}

material groups. Thereafter, the peak drawing force remained for a period and decreased until the end of the drawing process. Moreover, due to the stored strain energy in the drawn cups, the drawing force at the end of the process was not zero. Therefore, after the drawing process, the drawn cups underwent springback and stored stain energy released gradually. Moreover, the higher the residual drawing force is, the more significant the springback will be.

Table 3 lists the average geometrical values of drawn cups for each material group and their definitions are shown in Figure 5. Due to the limitation of the die, the blank wrinkled towards the punch. Therefore, the outer surface of the drawn cups was relatively smooth and the wrinkles were mainly on the inner surface of the drawn cups. Correspondingly, the difference between outer and minimum inner diameters was defined as the maximum distance, and the deviation between outer and maximum inner diameters was defined as minimum distance. Then, their relative difference, calculated from Equation (1), was employed as a judgement of wrinkles. With the increase of grain size, wrinkling phenomenon became significant. This is because that a few grains on the thickness direction make the deformability decrease, and consequently, compression stability on the flange of the blank is weak. Therefore, the blank is easy to wrinkle and needs great wrinkles to compensate this compression instability.

The thickness was measured based on the colourful images from a 3D profile microscope. A 3D structure of a drawn cup was combined from a serial of images at different focusing heights. Moreover, the drawn cup was coloured according to the height, and therefore the drawn cup's mouth can be distinguished based on the colour, as shown in Figure 5. Thus the thickness was tested and the average thickness was displayed in Figure 6. In addition, the wrinkled area was avoided for the thickness measurement. Therefore, the thickness was between the maximum and minimum distance for each material group. As there are limited grains on the drawn cups' mouth, thickness was determined by these few grains with different mechanical properties. As a result, there was no obvious trend regarding the average thickness at the cup mouth due to the randomness of grains properties and grain distributions.

$$
\text { Wrinkles }=\frac{\text { Max distance }- \text { Min distance }}{\text { Max distance }} \times 100 \%
$$

\section{Simulation}

Due to the axisymmetric geometry, a quarter of model was built to accelerate computational speed, and corresponding symmetric boundary conditions were applied on the straight edges of the blank. All the geometric parameters were the same as that of the real MDD system, and the parameters of material properties were obtained from the aforementioned micro tensile tests. All parts in this model were of shell elements including a deformable blank following the fully integrated elemental formulation and other three rigid parts with the BelytschkoTsay (BT) elemental formulation. Figure 7 displays (a) the MDD model, (b) the Voronoi blank model and (c) a drawn cup, respectively. In the Voronoi model, each Voronoi cell represented one grain, and they had similar size to the general grain size on the blank. All these grains were categorised into five groups, and each group's material properties were different from each other, while in accordance with the micro tensile test results. Moreover, commonly used three-parameter-barlat 

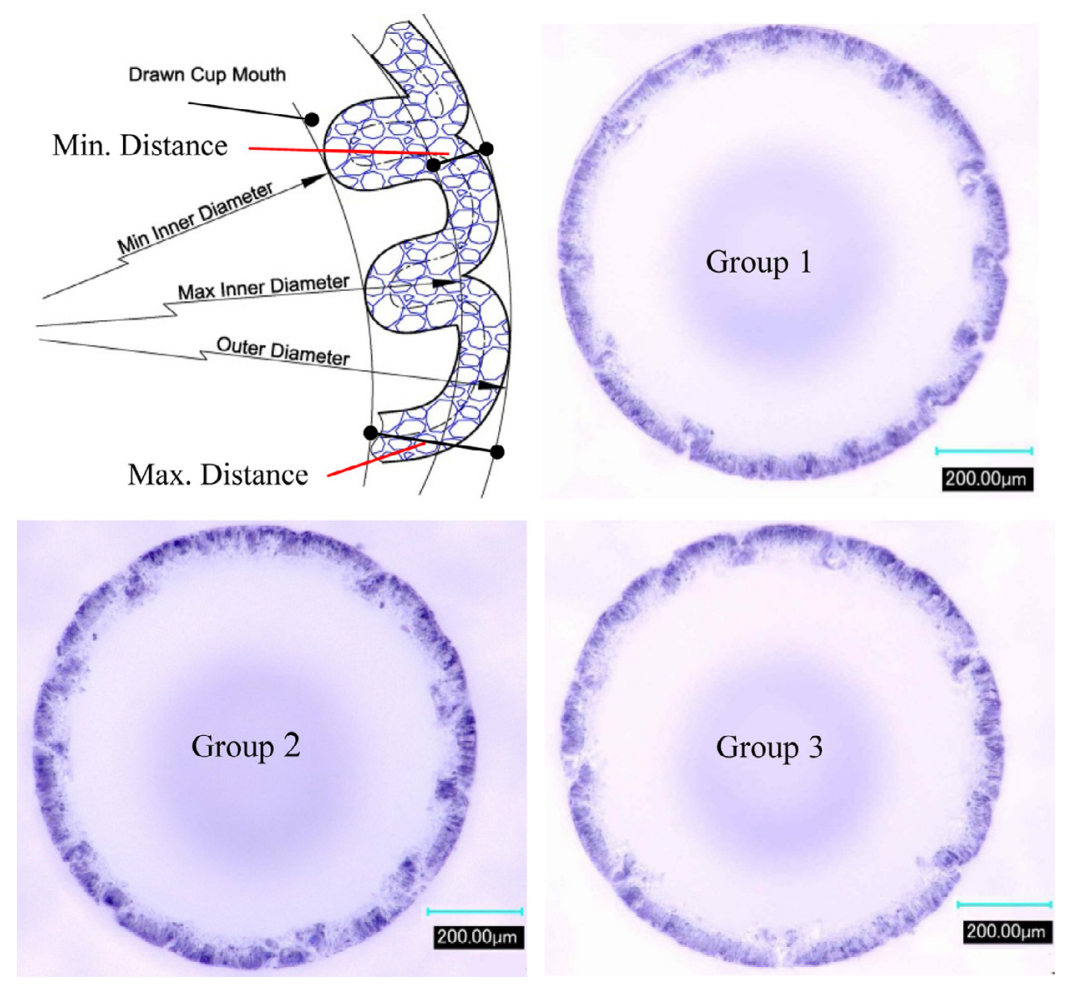

Figure 5. Definitions for wrinkling parameters and top views of drawn cups with different materials.

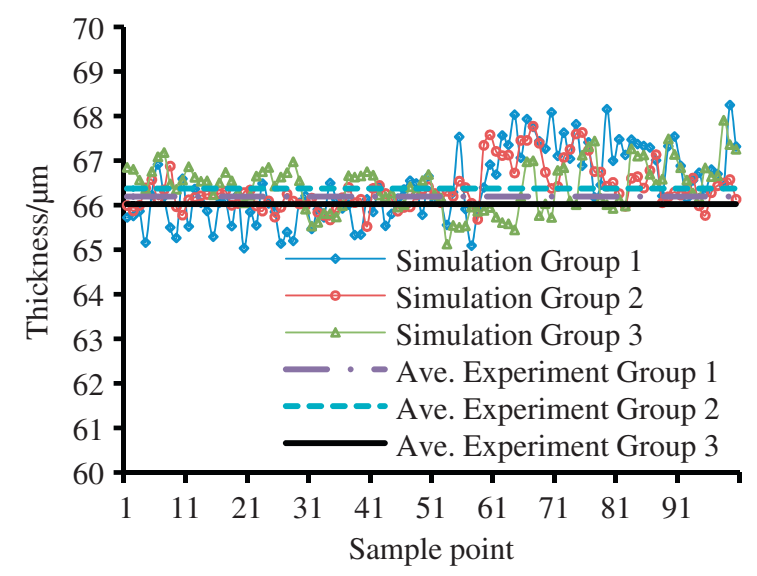

Figure 6. Thickness distribution at the drawn cups mouth.

material model was employed for the blank. Further, a surfaceto-surface contact algorithm specialised for forming process in LS-DYNA was adopted for monitoring contact behaviours between the blank and other rigid parts. Furthermore, the friction coefficients for each material group were tested by a friction tester. The MDD model was calculated explicitly, and then the drawn cup was exported for a subsequent implicit springback simulation in LS-DYNA. Due to the axisymmetric shape, one point on each symmetric boundary was fixed to limit rigid motion of the drawn cup. Moreover, the cup's material and elemental information were identical to that in the former explicit simulation. All the simulation models were run on a high performance computing (HPC) cluster in the University of Wollongong. Finally, simulation results can be compared with the data obtained from the experiments.

Drawing forces from the simulation are shown in Figure 4 and thickness distribution at the mouth of the drawn cups is presented in Figure 6. The drawing forces in simulation had a similar developing trend to that of experiments, while the peak values occurred earlier than the experimental ones. Furthermore, once the drawing force reached a peak value in simulation, the drawing force decreased until the end of the drawing process. Moreover, the residual forces were quite close for the simulation and experiments. Regarding the thickness at the drawn cups' mouth, due to the introduction of the Voronoi model, thickness was uneven and therefore different from that of a normal blank model with almost equal thickness at each node.

\section{Comparison}

The simulation and experimental results were compared. The peak drawing forces in experiments occurred earlier than simulation results. That lies in that randomly distributed grains with different mechanical properties on the blank cannot identically represent actual blank deformation behaviour and resulted in deviation in strain hardening effect in the simulation model. According to Equation (2) [13], flow stress, $\bar{\sigma}_{i}$, increased due to the strain hardening, whereas radial strain, $\ln \frac{R_{t}}{r_{0}}$, decreased with the drawing process. As the radial tension is a primary force of the drawing force, the two factors of radial stress determines the development of the drawing force. Moreover, the geometry utilised in the simulation model was 
(a)

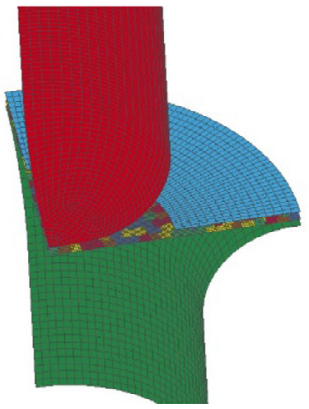

(b)

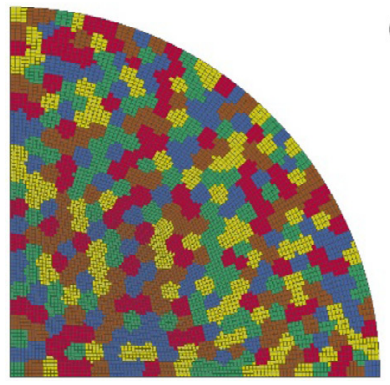

(c)

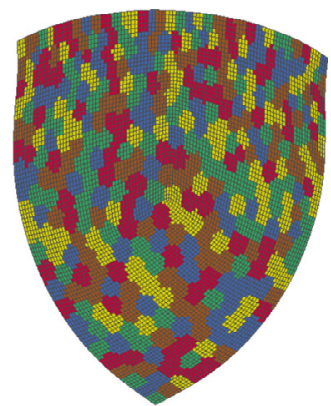

Figure 7. (a) MDD simulation model, (b) Voronoi blank model and (c) drawn cup.

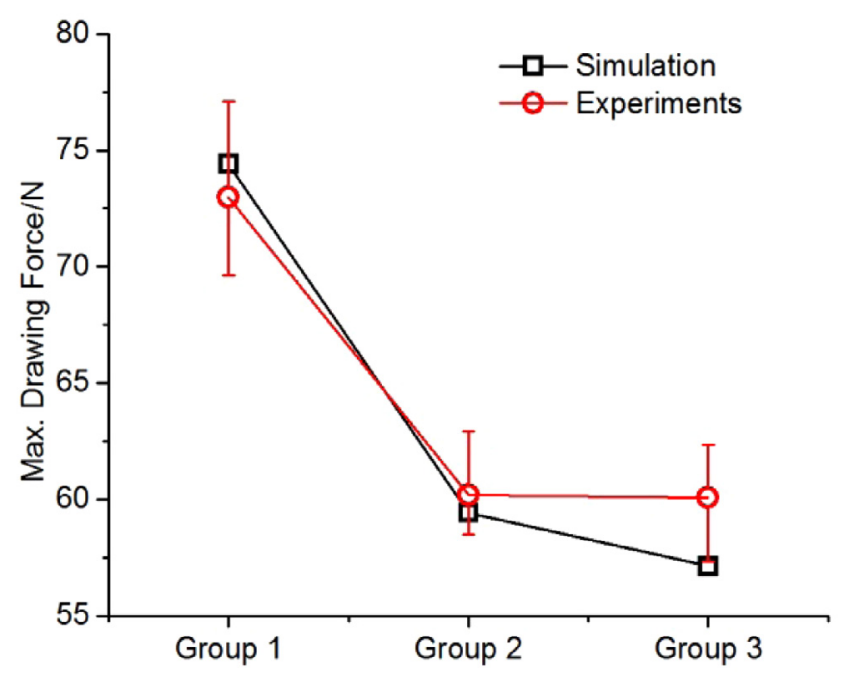

Figure 8. Maximum drawing forces.

the designed structure, whilst the real MDD machine may have tolerance to the design. Therefore, the difference in geometry also introduced the early occurrence of the peak drawing force. However, the peak drawing forces in the experiments and the simulation were close to each other for each material group, as shown in Figure 8. The material properties from the micro tensile tests considered the size effects and all the parameters used in the Voronoi model were from the micro tensile tests. Therefore, the peak drawing forces were close for the simulation and the experiments. The difference of the drawing force comes from the randomness of grain's distribution on the blank in the experiments and on the Voronoi model.

$$
\sigma_{\operatorname{rmax}}=1.1 \bar{\sigma}_{i} \ln \frac{R_{t}}{r_{0}}
$$

where $\sigma_{\text {rmax }}$ is the maximum radial stretch stress, $\bar{\sigma}_{i}$ is the average flow stress, $R_{t}$ is the current radius of the blank and $r_{0}$ is the inner radius of flange on the blank.

The peak drawing force in the experiments for each material group lasted for a certain period. This is because the balance between the resistance of deformation and the friction. According to Equation (2), the deformation resistance decreased while the friction force increased due to the thickened and wrinkled area of the blank entering into the gap between the punch and the die. This thickened and wrinkled area was drawn into the die and experienced an ironing-like process. By contrast, in the simulation, the penalty based friction measurement cannot present the increased friction force caused by the ironing. Therefore, once reaching the peak value, the drawing force decreased. After the ironing process, the drawing force in the experiments dropped faster than that in the simulation. As the thick area in the simulation was gradually squashed due to that the wrinkles was treated as thickening area, the drawing force decreased slowly. However, the residual forces were close for the simulation and the experimental results.

The average wall thickness at the cup mouth in simulation was quite close to that of experimental one. With different mechanical properties of different elements, the thickness in each element was different. While all the mechanical properties were obtained from the tensile tests, the general thickness in simulation was therefore in accordance with the experimental one.

\section{Conclusions}

The material properties considering size effects can be obtained through the developed micro tensile test system, and the micro deep drawing can be successfully conducted by the one-stroke blanking-drawing MDD system.

The heat treatments effectively change the material's microstructures (grain sizes) and mechanical properties. Correspondingly, the flow stress and the total elongation increase with the increase of heat treatment temperatures. Furthermore, the peak drawing force increases according to the heat treatment temperatures, whereas the wrinkling increases with the growth of the grains caused by the high heat temperature.

The Voronoi blank model can successfully simulate the blank on a grain scale in the micro deep drawing process. The peak drawing and the residual drawing force are in accordance with the experimental results. Furthermore, the thickness at the drawn cups mouth has a good agreement with the experimental one.

\section{Implications and influences}

This paper introduces the new micro tensile test system and the micro deep drawing system, which are beneficial to further investigation of micro forming processes with consideration of size effects. Furthermore, the developed MDD simulation 
model, especially the Voronoi model, is helpful for the further investigation and optimisation of micro forming processes.

Acknowledgements. The first author greatly thanks for the financial support from the Chinese Scholarship Council (CSC, No. 061620120011) and the International Postgraduate Tuition Award (IPTA) from the University of Wollongong. The support from the Australian Research Council (ARC) for Future Fellowship and the Japan Society for Promotion of Science (JSPS) for Invitation Fellowship (Zhengyi Jiang) is also appreciated.

\section{References}

1. Q. Yi, A. Brockett, Y. Ma, A. Razali, J. Zhao, C. Harrison, W. Pan, X. Dai, D. Loziak, Int. J. Adv. Manuf. Technol. 47 (2010) 821-837.

2. K. Manabe, T. Shimizu, H. Koyama, M. Yang, K. Ito, J. Mater. Process. Tech. 204 (2008) 89-93.

3. J. Xu, B. Guo, D. Shan, C. Wang, J. Li, Y. Liu, D. Qu, J. Mater. Process. Technol. 212 (2012) 2238-2246.
4. L. Peng, P. Yi, P. Hu, X. Lai, J. Ni, J. Micro Nano-Manuf. 3 (2015) 11006.

5. Z. Xu, L. Peng, X. Lai, M. Fu, Mat. Sci. Eng. A-Struct. 611 (2014) 345-353.

6. F. Vollertsen, Key Eng. Mat. 473 (2011) 3-12.

7. Z. Fang, Z. Jiang, X. Wang, C. Zhou, D. Wei, X. Liu, Int. J. Adv. Manuf. Technol. 79 (2015) 1905-1914.

8. T. Furushima, H. Tsunezaki, K.-I. Manabe, S. Alexsandrov, Int. J. Mach. Tools Manuf. 76 (2014) 34-48.

9. A. Molotnikov, R. Lapovok, C.F. Gu, C.H.J. Davies, Y. Estrin, Mat. Sci. Eng. A-Struct. 550 (2012) 312-319.

10. N. Ma, X.H. Dong, J. Shanghai Jiaotong Univ. 12 (2007) 323-327.

11. H.N. Lu, D.B. Wei, Z.Y. Jiang, X.H. Liu, K. Manabe, Comp. Mater. Sci. 77 (2013) 44-52.

12. L. Luo, Z. Jiang, H. Lu, D. Wei, K. Linghu, X. Zhao, D. Wu, Procedia Eng. 81 (2014) 2409-2414.

13. J. Hu, Z. Marciniak, J. Duncan, Mechanics of sheet metal forming, Butterworth-Heinemann, Oxford, 2002. pp. 117-127.

Cite this article as: Luo L, Jiang Z, Wei D, Manabe K-I, Sato H, He X \& Li P: An experimental and numerical study of micro deep drawing of SUS304 circular cups. Manufacturing Rev. 2015, 2, 27. 